\section{DEVELOPMENT}

\section{Hedgehog proliferation}



reception of the Hh signal might be needed for entry to $S$ phase. To test this, the authors looked at what would happen if Hh signalling was blocked during eye development. They found that second mitotic wave cells with mutated smoothened (smo), a gene that is required for $\mathrm{Hh}$ signalling, do not enter $S$ phase. By interruptus $(\mathrm{Ci})$ - the transcription factor that mediates Hh signalling - drove G1-arrested cells to enter $S$ phase.

is Cyclin D. During eye

elopment, the highest expression Cyclin D overlaps with that of the expression of Cyclin $D$ ? Support for this idea came from the observation that levels of Cyclin D are reduced in smo-mutant clones, an of Cyclin D entering $S$ phase, indicated that
As well as promoting entry into $S$ phase, Cyclin D induces cell growth. $\mathrm{Du}$ and co-workers therefore wondered whether $\mathrm{Hh}$ might also regulate growth, so they studied the effects of overexpressing either $\mathrm{Ci}$ or Patched (Ptch; an inhibitor of $\mathrm{Hh}$ signalling) in clones of undifferentiated wing-disc cells. Whereas Ptch overexpression clones were considerably smaller than controls, Ci overexpression clones were much larger, which indicates that Hh signalling not only promotes $\mathrm{S}$ phase, but that it also regulates cell growth.

Cyclin $\mathrm{E}$ also promotes $\mathrm{S}$ phase, and reduced or increased levels of this protein could be detected with loss of Smo or overexpression of $\mathrm{Ci}$, respectively. The authors then looked at how $\mathrm{Hh}$ signalling might induce the transcription of Cyclin E. They identified several sequences in the Cyclin E promoter with homology to the consensus Cibinding site, and used chromatin immunoprecipitation to show that

\title{
A deadly combination
}

In some cell lines, an epithelialmesenchymal transition (EMT) arises as the result of a joint effort between Hras and transforming growth factor- $\beta$ (Tgf- $\beta$ ). How relevant this is to the multistage nature of in vivo tumour progression, though, is a burning question.

So, Allan Balmain's group studied whether changes in the levels of Hras and Tgf- $\beta$ affect tumour progression, using a series of wellcharacterized tumour cell lines that arise from initiated cells that carry activating mutations in the Hrasl gene. And, as they now report in Nature Cell Biology, Smad2 (a downstream target of Tgf- $\beta$ signalling) and Hras surpass discrete thresholds during progression from early-stage papillomas, through squamous carcinomas, to late-stage undifferentiated spindle-cell tumours.

First, the authors studied the molecular changes that occur when squamous carcinomas are converted into spindle-cell tumours. Tgf- $\beta$-mediated transcriptional activity was very high in the spindle cells, and phosphorylated Smad2 accumulated in the nucleus, which indicated that the Tgf- $\beta$ pathway was activated in these cells. Furthermore, in primary material from spindle-cell tumours, but not from differentiated tumours or squamous carcinomas, Smad2 was phosphorylated and predominantly localized in the cytoplasm.

Although Smad2 alone induced changes in the migration of squamous carcinoma cells, only in the presence of increased levels of mutated Hras did changes in cell shape and the expression of genes such as $\alpha$-smoothmuscle actin (a mesenchymal marker) occur, resulting in EMT.

The authors then investigated whether, once this stage has been reached, Tgf- $\beta$ signalling by Smad 2 is still necessary for tumour progression. Expression of a dominant-negative form of Smad 2 showed that this is indeed the case; spindle cells that expressed this construct reverted to a more epithelial phenotype and took on many features of epithelial gene expression. Notably, surface expression of $\alpha v \beta 3$ integrin was lost, and this was coincident with the loss of collagen-matrix invasion. In vivo, this correlated with an inability to form tumours. By contrast, parental spindle cells or spindle cells that express a dominant-active form of Smad2 formed tumours, and those formed by dominant-active Smad 2 were particularly invasive. Expression of dominant-active Smad2 also promoted extravasation into the target tissue, and a subsequent increase in lung metastases.

As the ability of a tumour to metastasize is the main determinant of whether or not patients with cancer die of their disease, these findings that different thresholds of Hras and Tgf- $\beta$ activity — intermediate levels of Smad2 co-operating with Hras to induce EMT and invasiveness, and even higher levels of Smad 2 being required for metastasis - are crucial for metastasis offer the opportunity for the design of small-molecule inhibitors to prevent the spread of tumours.

Katrin Bussell, Associate Editor, Nature Reviews Molecular Cell Biology

(2) References and links ORIGINAL RESEARCH PAPER Oft, M., Akhurst, R. J. \& Balmain, A. Elevated levels of activated Smad2 and Hras control epithelial-mesenchymal transformation, tumour cell extravasation and metastasis. Nature Cell Biol. 4, 487-494 (2002) FURTHER READING Frame, S. \& Balmain, A. Integration of positive and negative growth signals during ras pathway activation in vivo. Curr. Opin. Genet. Dev. 10, 106-113 (2000) | Akhurst, R. J. \& Derynck, R. TGF- $\beta$ signaling in cancer - a double-edged sword. Trends Cell Biol. 11, S44-S51 (2001) WEB SITE

Allan Balmain's lab: http://cc.ucsf.edu/balmain 